physics, The Czech Academy of Sciences, Královopolská 135, 61265 Brno, CZ

357550@mail.muni.cz

The chromatin organization of ribosomal genes, which encode essential 25S, $18 \mathrm{~S}$ and 5.8S ribosomal RNA, remains unresolved to this day in many biological models. Hundreds of rDNA copies exist in the genome in a mostly tandem, head-to-tail arrangement. Nucleoli, the main transcription factories of the cell, are known to assemble around actively transcribed rDNA copies. Evidence from electron microscopy studies shows a subset of actively transcribed genes which appear mostly nucleosome-free [1], but this represents only a small fraction of rDNA units. Our understanding of the epigenetic state of the remaining copies is still incomplete [2]. Our main goal was to identify individual rDNA chromatin fibers and probe their chromatin structure. Methods We prepared chromatin fibers from the plant model Arabidopsis thaliana in combination with immunofluorescence and FISH detection of histone marks in rDNA loci. Results We show the presence of histones and histone modifications in rDNA clusters, even in plants with a reduced number of rDNA copies [3]. This indicates that a subset of rDNA copies exists in a poised state for transcription, marked by the presence of $\mathrm{H} 3 \mathrm{~K} 4 \mathrm{me} 3$ and $\mathrm{H} 3 \mathrm{~K} 27 \mathrm{me} 3$ modifications. Furthermore, these histone modifications are found intermingling or colocalizing on individual chromatin fibers in plants, reinforcing the notion that as opposed to mammalian models, H3K27me3 is not a marker of compact heterochromatin in plants. Conclusions: Microscopy of chromatin fibers has been used previously to study the structure of centromeres. We show that it is possible to adapt this strategy to study the epigenetic structure of rDNA. Once optimized, this method can be used to study repetitive genomic regions, telomeres and satellites included, and fill the gaps in knowledge in genomic regions hard to analyze by ChIP-seq.

Fundings: This project was supported by The Czech Science Foundation, grant number 19-11880Y and Ministry of education, youth and sports INTER-COST LTC18048.

References: 1. Miller OL, Beatty BR. 1969. Visualization of Nucleolar Genes. Science, 164: 955-957. 2. Dvořáčková M, Fojtová M, Fajkus J. 2015. Chromatin dynamics of plant telomeres and ribosomal genes. Plant J. 83:1837. 3)Pavlištová V, Dvořáčková M, Jež M, Mozgová I, Mokroš P, Fajkus J. 2016. Phenotypic reversion in fas mutants of Arabidopsis thaliana by reintroduction of FAS genes: variable recovery of telomeres with major spatial rearrangements and transcriptional reprogramming of $45 \mathrm{~S}$ rDNA genes. Plant J. 88: 411-424.

doi: http://dx.doi.org/10.7124/bc.0009DF

\section{G-1. Large-scale chromatin remodelling and transcriptional deregulation on der11 following translocation in Mantle Cell Lymphoma}

Diego Germini $^{1,2}$, Fatimata Bintou Sall ${ }^{1,2}$, Diana Markozashvili1 ${ }^{1,2}$, Andrei Pichugin ${ }^{1,2}$, Valérie Camara-Clayette ${ }^{3}$, Vincent Ribrag ${ }^{3}$, Marc Lipinski ${ }^{1,2}$, Yegor Vassetzky ${ }^{1,2}$

${ }^{1}$ UMR 8126, Paris Saclay University, Paris-Sud University, Institut Gustave Roussy, CNRS, Villejuif, 94805, France; ${ }^{2}$ LIA 1066 French-Russian Joint Cancer Research Laboratory, 94805 Villejuif, France - 119334 Moscow, Russia; ${ }^{3}$ Institut de Cancérologie Gustave-Roussy, Villejuif, France germinidiego@gmail.com

Mantle cell lymphoma (MCL) is an aggressive B-cell non-Hodgkin lymphoma characterized 
by poor prognosis and survival rate. Its genetic hallmark is the translocation $\mathrm{t}(11 ; 14)$ which leads to the overexpression of cyclin D1 (CCND1) gene which becomes juxtaposed to the immunoglobulin heavy chain (IGH) gene on the newly formed der14 chromosome. This recurrent feature is however not sufficient to promote the development of the disease as expression of CCND1 under different known IgH enhancers in transgenic mice is not sufficient for tumor development. Additional alterations are necessary to develop a malignant phenotype. When a translocation occurs, it can induce overall nuclear reorganization, epigenetic changes and altered gene expression that may contribute to oncogenesis. Here we investigated changes in nuclear positioning of gene loci and their transcription after the $t(11 ; 14)$ focusing our attention on the events occurring on the der11 chromosome. Methods. 3D-immunoFISH and image analysis software were used to analyze gene loci position in nuclear space. To analyze changes of transcriptional level of genes located on the der11, quantitative RT-PCR, bioinformatic analysis and data mining were performed. ChIP was carried out to analyze specific interactions between nucleolin and the genome in MCL. Results. We demonstrated that the expression of many genes located close to the translocation breakpoint was deregulated in MCL compared to other lymphomas and to B-cells from healthy donors. Most of these genes were located on the der11 after the $\mathrm{t}(11 ; 14)$. We found that the der11 is relocated in close proximity to the nucleolus. Here the nucleolin, that is part of the transcriptional factor LR-1 can deregulate gene expression by direct binding to promoters. We found that the LR-1 consensus sequence and the nucleolin binding sites are significantly enriched in the regions covered by the deregulated genes compared to the rest of chromosme11 and to cells without the $t(11 ; 14)$. Conclusions. We identified new epigenetic events that contribute to MCL development following $\mathrm{t}(11 ; 14)$.

doi: http://dx.doi.org/10.7124/bc.0009E0

\section{G-2. Histone deacetylase inhibitor obstructs non- homologous end joining DNA repair in oncogene-transformed but not in normal cells}

Olga Gnedina, Alisa Morshneva, Svetlana Svetlikova, Maria Igotti

Institute of Cytology, Russian Academy of Science olga.o.gnedina@gmail.com

Histone deacetylase inhibitors (HDACi) suppress the growth of tumor cells due to the induction of cell cycle arrest, senescence or apoptosis (1-2). There are a few data concerning the reduction of DNA repair capability by HDACi in tumor cells (3-4). Previously we established that HDACi-induced G1/S cell cycle arrest of E1A+Ras-transformed mouse embryonic fibroblasts was accompanied with an accumulation of the marker of damaged DNA - $\gamma \mathrm{H} 2 \mathrm{AX}$ foci (5). Among all DNA damages, the double-strand breaks (DSB) are the most crucial for a cell. Since non-homologous end joining (NHEJ) is a principal repair mechanism of DSB whatever of the cell cycle phase, we focused our research on this repair mechanism. The aim of this study is to compare the DNA repair efficiency in transformed and normal cells after HDACi treatment. 